\title{
Antiferromagnetism and phase separation in electronic models for doped transition-metal oxides
}

\author{
Shun-Qing Shen and Z. D. Wang \\ Department of Physics, The University of Hong Kong, Pokfulam Road, Hong Kong
}

(Received 12 March 1998; revised manuscript received 13 July 1998)

\begin{abstract}
We investigate the ground state properties of electronic models for doped transition-metal oxides. An effective $t-J$ like Hamiltonian is derived from the case of strong Hund coupling between the conduction electrons and localized spins by means of the projection technique. An attractive interaction for conduction electrons and an antiferromagnetic coupling of the localized spin are obtained. A large ratio of the attraction to effective electron hopping, which is modulated by the spin background, will lead to the phase separation. The antiferromagnetic phase and the phase separation appear in the case of either high or low density of electrons. The possible relevance of the phase separation to the charge stripe phase in doped transition-metal oxides is discussed. [S0163-1829(98)51238-6]
\end{abstract}

The problem of doped Mott insulators has attracted much attention because of its relevance to high temperature superconductivity and the colossal magnetoresistance effect. Recent experiments of doped lanthanum cuperate, ${ }^{1}$ nickelate ${ }^{2}$ and manganite ${ }^{3}$ families of materials exhibit a different type of charge ordering and spin ordering in an extensive region. For example, the charge and spin stripe phases were observed in $\mathrm{La}_{2-x} \mathrm{Sr}_{x} \mathrm{NiO}_{4}$ samples. ${ }^{4}$ Along the charge stripe, there is strong antiferromagnetic correlation. It is also shown experimentally that the charge ordering collapses in the presence of an external magnetic field, which can destroy antiferromagnetic ordering. ${ }^{5}$ Many efforts have been devoted to understand the origin of the phenomena and its intrinsic relevance to various anomalous transport properties.

In this paper, starting from an electronic model for doped transition-metal oxides, we derive an effective $t$ - $J$ like Hamiltonian for the case of strong Hund coupling. An attractive interaction between conduction electrons, which is associated with the antiferromagnetic correlation, is obtained. Both the attraction and electron hopping are modulated by the configuration of two localized spins on the nearestneighbor sites. A larger ratio between them will lead to the phase separation, which is expected in terms of the ideas of frustrated phase separation for the charge stripe phase. ${ }^{6}$ An antiferromagnetic background leads to attraction between electrons. We find that the phase separation with electronrich and electron-poor regimes has a lower energy than an antiferromagnetic phase with a uniform density of charge. A phase diagram for the model is presented. The possible relevance to the phase separation and the charge stripe phases in doped lanthanum manganites and nickelates are also discussed.

An electronic model to describe doped transition metal oxides is a Kondo-like lattice Hamiltonian with the strong Hund coupling

$$
H=-t \sum_{\langle i j\rangle, \sigma} c_{i, \sigma}^{\dagger} c_{j, \sigma}-J_{H} \sum_{i} \mathbf{S}_{i} \cdot \mathbf{S}_{i c}
$$

where $c_{i, \sigma}^{\dagger}$ and $c_{i, \sigma}$ are the creation and annihilation operators for conduction electrons, respectively. $\mathbf{S}_{i c}$ $=\Sigma_{\sigma, \sigma^{\prime}} \sigma_{\sigma, \sigma^{\prime}} c_{i, \sigma^{\prime}}^{\dagger} c_{i, \sigma^{\prime}} / 2$ is the spin operator for the conduc- tion electron and $\sigma$ are the Pauli matrices. $\mathbf{S}_{i}$ is the total spin of the localized electrons at site $i . J_{H}>0$ is the Hund coupling between the conduction and localized electrons. In the manganites, three $t_{2 g}$ electrons are almost localized and form an $S=3 / 2$ spin state according to the Hund rule. Electrons in $e_{g}$ orbital form a conduction band. ${ }^{7}$ In the nickelate, the localized spin is just $S=1 / 2 .^{8}$ In the case of a single electron the ground state is a fully saturated ferromagnet. It is easier for the conduction electron to move when the two localized spins on the nearest-neighbor sites are parallel to each other. The process may lead to metallic ferromagnetism, and is called the double exchange mechanism. ${ }^{9-11}$

Usually the Hund coupling is very large in either manganites or nickelates. An infinite $J_{H}$ limit is often taken in these systems, especially to investigate the double exchange ferromagnetic phase. However, in the limit, the spin of electron is completely frozen to localized spin to form an $S+1 / 2$ state due to the strong Hund coupling, and the model is reduced to a spinless fermion system without a direct electron-electron interaction, which cannot describe the charge ordering and antiferromagnetism. We consider the large and finite $J_{H}$ $(\gg t)$ case. As the strong Hund coupling forces most of electrons to form $S+1 / 2$ states with the localized spins, we will restrict our discussion in the space, which includes only the empty and single occupancies with $S+1 / 2$ state. The finite $J_{H}$ effect can be regarded as the perturbation correction to the large $J_{H}$ limit. The operator to project onto the space of the configurations with empty and the $S+1 / 2$ states is

$$
P=\prod_{i} P_{i}=\prod_{i}\left(P_{h i}+P_{s i}^{+}\right)
$$

where $P_{h i}=\left(1-n_{i, \uparrow}\right)\left(1-n_{i, \downarrow}\right)$ and

$$
\begin{aligned}
P_{s i}^{+} & =\sum_{\sigma, \sigma^{\prime}}\left(P_{i}^{+}\right)_{\sigma \sigma^{\prime}} \tilde{c}_{i, \sigma}^{\dagger} \tilde{c}_{i, \sigma^{\prime}} \\
& =\sum_{\sigma, \sigma^{\prime}}\left(\frac{\mathbf{S}_{i} \cdot \sigma+(S+1) \mathbf{I}}{2 S+1}\right)_{\sigma \sigma^{\prime}} \tilde{c}_{i, \sigma^{\prime}}^{\dagger} \tilde{c}_{i, \sigma^{\prime}}
\end{aligned}
$$

.


The operator $\tilde{c}_{i, \sigma}^{\dagger}=\left(1-n_{i,-\sigma}\right) c_{i, \sigma}^{\dagger}$ rules out double occupancy on the same site. $\mathbf{I}$ is a unity matrix. Utilizing the Schrieffer-Wolf transformation, ${ }^{12}$ a $t-J$ like effective Hamiltonian is derived

$$
H_{e f f} \approx-t \sum_{\langle i j\rangle} \bar{c}_{i, \sigma}^{\dagger} \bar{c}_{j, \sigma}+4 v_{0} \sum_{\langle i j\rangle}\left(\overline{\mathbf{S}}_{i c} \cdot \overline{\mathbf{S}}_{j c}-\frac{1}{4} \bar{n}_{i} \bar{n}_{j}\right),
$$

where $v_{0}=t^{2} /\left(J_{H} S\right), \overline{\mathbf{S}}_{i c}=\Sigma_{\sigma, \sigma^{\prime}}(\sigma)_{\sigma \sigma^{\prime}} \bar{c}_{i, \sigma^{\dagger}} \bar{c}_{i, \sigma^{\prime}} / 2$, and $\bar{c}_{i, \sigma}$ $=\Sigma_{\sigma^{\prime}}\left(P_{i}^{+}\right)_{\sigma \sigma^{\prime}} \tilde{c}_{i, \sigma^{\prime}}$. In Eq. (3), except for ignorance of higher-order perturbation correction and a constant term $-N_{e} J_{H} S / 2$ ( $N_{e}$ is the number of electrons), we also neglect three-site terms in order of $t^{2} / J_{H} S$ which describe indirect hopping process between the next-nearest-neighbor sites. They are of order $t / J_{H} S(\ll 1)$ when compared with the first, direct hopping term in Eq. (3). A detailed discussion including these terms will be published elsewhere. The physical meaning of the operator $\overline{\mathbf{S}}_{i c}$ is the component of electron spin along the localized spin on the same site meanwhile the electron and localized spin form an spin $S+1 / 2$ state. It is shown that $(2 S+1) \overline{\mathbf{S}}_{i c}\left(=\mathbf{S}_{i}^{t}\right)$ is an spin operator with $S+1 / 2$ if the site is occupied by a single electron. Let us first consider two limits. When $J_{H} \rightarrow+\infty, v_{0} \rightarrow 0$. The model is reduced to the quantum double exchange model. ${ }^{13,14}$ Expanding the dressed operators $\bar{c}$ in Eq. (3), we find a direct exchange term for localized spins and its effective exchange coupling is approximately

$$
J_{d e} \approx-t \sum_{\sigma}\left\langle\tilde{c}_{i, \sigma}^{\dagger} \tilde{c}_{j, \sigma}\right\rangle /(2 S+1)^{2}
$$

where $\langle\cdots\rangle$ represents the average of the ground state. The coupling is proportional to the kinetic energy and is always ferromagnetic. It reaches its minimum at quarter filling $(\rho$ $=1 / 2$ ) and vanishes at two density limits $\rho=0$ and 1 in a sense of the mean-field approximation. This result is consistent with the physical picture of the double exchange ferromagnet. At half filling, which means that the number of electrons is equal to the number of lattice sites, each site is occupied by one electron and there is no empty site. The effective Hamiltonian (3) is reduced to an antiferromagnetic Heisenberg model with spin $S+1 / 2$

$$
H_{A F}=\frac{4 v_{0}}{(2 S+1)^{2}} \sum_{\langle i j\rangle}\left(\mathbf{S}_{i}^{t} \cdot \mathbf{S}_{j}^{t}-\left(S+\frac{1}{2}\right)^{2}\right) .
$$

This is consistent with rigorous results of the model (1) at half filling for any $J_{H}$ that the ground state is spin singlet on a hypercubic lattice. ${ }^{15}$ In the antiferromagnetic Heisenberg model, it is shown rigorously that the ground state possesses antiferromagnetic long-range order on a square lattice for spin 1 or higher and cubic lattice for spin $1 / 2$ or higher. ${ }^{16}$ Therefore, here as $S+1 / 2 \leqslant 1$, we conclude that the Kondo lattice model at half-filling and with strong Hund coupling possesses antiferromagnetic long-range order, which is completely opposite to the case of low density of electrons where ferromagnetic correlation is predominant.

Although the physics of the two terms in the Hamiltonian (3) is clear, the combination of the two terms makes it very complicated. The usual $t-J$ model from the large $U$ Hubbard model can be regarded as a specific case of $S=0$ with a finite $v_{0}$. Many efforts were attempted to investigate the antiferromagnetism and superconductivity. Emery et al. proposed that a Heisenberg antiferromagnet in a $t$ - $J$ model is always unstable to a phase separation at a sufficient dilute doping. ${ }^{17}$ Their conjecture is supported numerically at larger $J / t$, but it is still an open problem at small $J / t$ strength. ${ }^{18}$ Nevertheless, for the usual $t-J$ model, $J=4 t^{2} / U$ and should be very small in a physical region. The localized spin in $J$ term is $1 / 2$. In our case, the spin background can modulate the electronic behavior, and localized spin can be any value. To simplify our discussion, we take the classic spin approximation or large $S$ limit. The spin $\mathbf{S}_{i}$ can be parametrized by polar angles $\theta_{i}$ and $\phi_{i}$ and $S /(2 S+1) \approx 1 / 2 . \quad \mathbf{S}_{i}=S \vec{s}_{0 i}$ and $\vec{s}_{0 i}$ $=\left(\sin \theta_{i} \cos \phi_{i}, \sin \theta_{i} \sin \phi_{i}, \cos \theta_{i}\right)$. Except for the exchange coupling between the conduction electron and localized spin, it is believed that the antiferromagnetic exchange coupling between the localized spins $J_{A F} \mathbf{S}_{i} \cdot \mathbf{S}_{j}$ also plays an essential role in determining the phase diagram of manganites, especially in the region of low density of electrons. The projected operator $\overline{\mathbf{S}}_{i}=P_{i} \mathbf{S}_{i} P_{i}=S \vec{S}_{0 i}$. We shall consider it in our following discussion. In this approach, the quantum $t-J$ model including the localized spin coupling is reduced to

$$
\begin{aligned}
H_{r d e}= & -t \sum_{\langle i j\rangle} c_{i j} \alpha_{i}^{\dagger} \alpha_{j}-2 v_{0} \sum_{\langle i j\rangle} \sin ^{2} \frac{\Theta_{i j}}{2} \alpha_{i}^{\dagger} \alpha_{i} \alpha_{j}^{\dagger} \alpha_{j} \\
& +J_{A F} S^{2} \sum_{\langle i j\rangle} \cos \Theta_{i j},
\end{aligned}
$$

where $\alpha_{i}=\cos \theta_{i} / 2 \tilde{c}_{i, \uparrow}+\sin \theta_{i} / 2 e^{-i \phi_{i}} \tilde{c}_{i, \downarrow}$

$$
c_{i j}=\cos \frac{\theta_{i}}{2} \cos \frac{\theta_{j}}{2}+\sin \frac{\theta_{i}}{2} \sin \frac{\theta_{j}}{2} e^{-i\left(\phi_{i}-\phi_{j}\right)}
$$

$\cos \Theta_{i j}=\cos \theta_{i} \cos \theta_{j}+\sin \theta_{i} \sin \theta_{j} \cos \left(\phi_{i}-\phi_{j}\right)$.

$\Theta_{i j}$ is the angle between the two spin units $\vec{s}_{0 i}$ and $\vec{s}_{0 j}$ and $\left|c_{i j}\right|=\cos \left(\Theta_{i j} / 2\right)$. $\alpha$ operators are for the conduction electrons whose spins are frozen by the localized spins on the same site, and therefore can be considered only to describe the charge degrees of freedom. The first part of $H_{r d e}$ is the usual double exchange model with a Berry phase, and the second part comes from the correction of the finite $J_{H}$. Both the renormalized coefficients $c_{i j}$ of hopping terms and $\sin ^{2}\left(\Theta_{i j} / 2\right)$ of the density-density interaction depend on the background of the spin configurations. Our following discussion will be based on the Hamiltonian (5).

From the point of view of localized spins, the mobile electrons favor to the ferromagnetic correlation. However, the finite $J_{H}$ as well as the direct exchange coupling $J_{A F}$ tends to form antiferromagnetism. For instance, in the onedimensional case, the effective double exchange coupling is approximately proportional to $-\sin \rho \pi / \pi$. It reaches its minimum at quarter filling and approaches to zero at two end limits. The exchange coupling from the finite $J_{H}$ is approximately proportional to $\rho^{2}$. It is stronger than the double exchange coupling at a higher density of electrons, but is weaker at a lower density of electrons. As far as the direct exchange coupling $J_{A F} S^{2}$ is introduced, the double exchange coupling is always suppressed at the two end limits of density. If $J_{A F} S^{2}$ is sufficiently large, the ferromagnetic phase is 


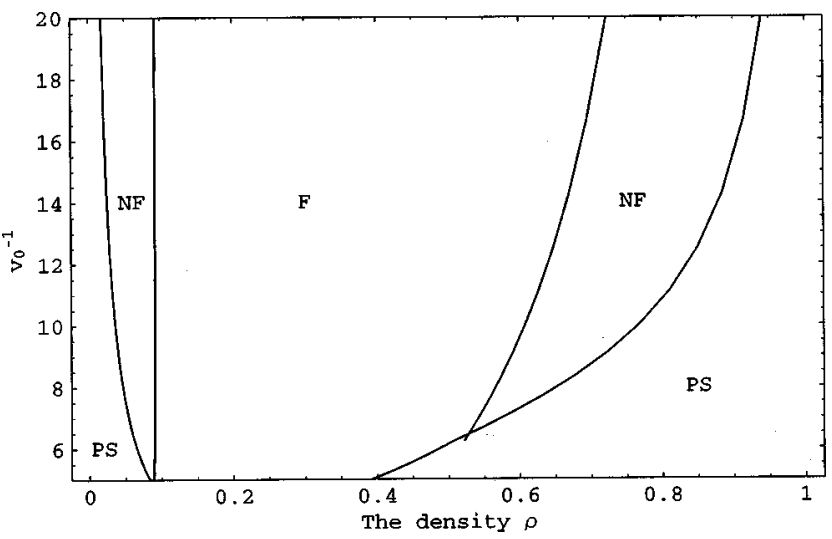

FIG. 1. The phase diagram for a square lattice at $J_{A F} S^{2}=0.05$. " $F$ ' means ferromagnetic. In that regime it is a metallic double exchange ferromagnet. "PS" means the phase separation on an antiferromagnetic background. " $\mathrm{NF}$ ' is between the ferromagnetic phase and phase separation. It is paramagnetic or a mixture of some noncollinear magnetism from the point of view of the mean field theory.

always unstable. If $J_{A F} S^{2}$ is not sufficiently large the double exchange ferromagnetism can survive in a finite range of doping. A possible phase diagram is shown in Fig. 1 for the chosen parameters. As $\left|c_{i j}\right|$ is proportional to $\cos (\Theta / 2)$, not $\cos \Theta$ for the ferromagnetic coupling, it is also possible to lead some noncollinear magnetism. ${ }^{11,14,19}$ The boundaries of phases in Fig. 1 and later in Figs. 2 and 3 are determined by comparing the ferromagnetic phase with the canted ferromagnetic phase, the spin spiral phase, the antiferromagnetic phase, and the phase separation in a mean-field approach.

From the point of view of conduction electrons, the hopping of electrons is heavily dragged by the spin background. The hopping is prohibited when the angle $\Theta_{i j}=\pi$. The effective interaction is also determined by $\Theta_{i j}$ as well as $v_{0} / t$. The ratio $-\left[2 v_{0} \sin ^{2}\left(\Theta_{i j} / 2\right)\right] /\left[t \cos \left(\Theta_{i j} / 2\right)\right]$ approaches to zero when $\Theta=0$, i.e., the ferromagnetic case, and becomes divergent when $\Theta=\pi$, i.e., the antiferromagnetic case. The consequence is quite different from the usual $t-J$ model, in which the ratio $J / t$ is fixed and is usually very small. When localized spins form a fully saturated ferromagnet, the conduction electrons are a spinless free fermion gas. Oppositely when the localized spins form an antiferromagnetic background, the attraction becomes rather strong since the hop-

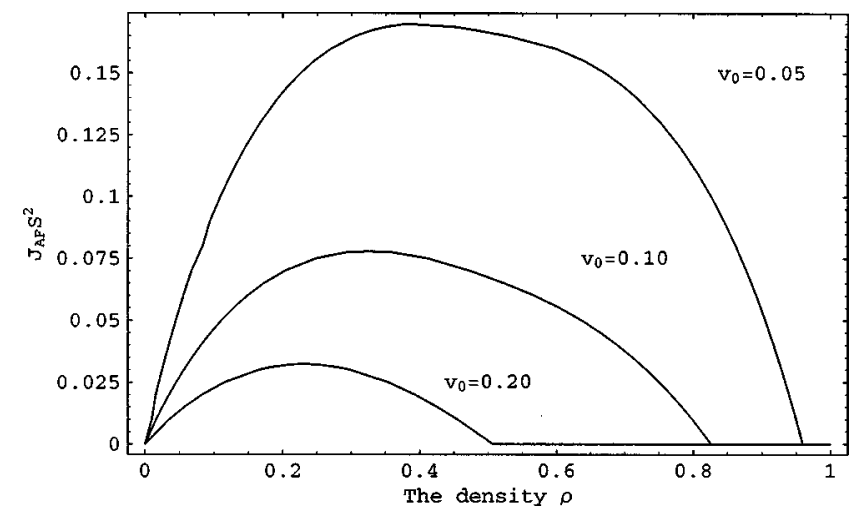

FIG. 2. The density dependence of the critical value of $J_{A F} S^{2}$ to the phase separation for $v_{0}=0.05,0.10$, and 0.02 in a square lattice.

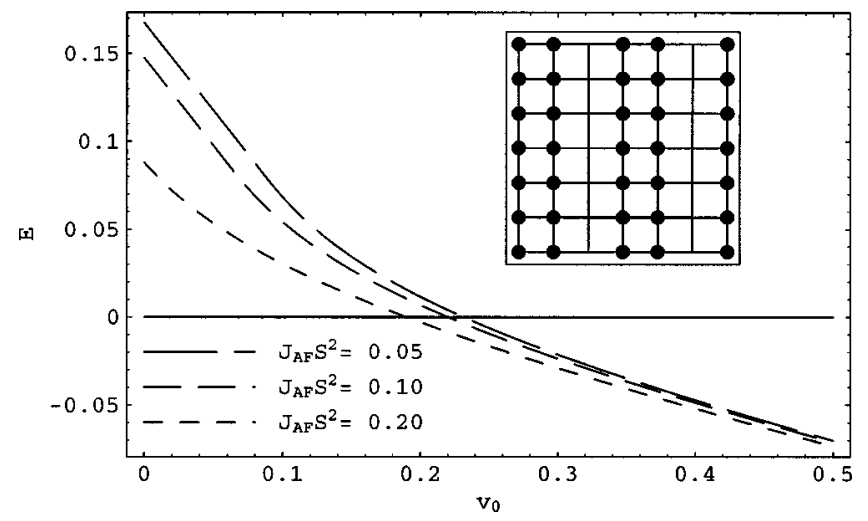

FIG. 3. The $v_{0}$ dependence of the energy difference, E, between the charge stripe and a state with a uniform density of charge for different $J_{A F} S^{2}$. The inset is the charge stripe phase we discuss. The black points stand for single occupancies of electrons.

ping of electrons is completely suppressed even for very small $v_{0} / t$. Strong attraction between fermions will lead to the instability to the phase separation. When the phase separation occurs, the system is divided into two parts: electronrich and electron-poor regimes. In the electron-rich regime, all electrons accumulate together and $\rho \rightarrow 1$. In this case, the kinetic energy vanishes and the average energy per bond is $-J_{A F} S^{2}-2 v_{0}$. In the electron-poor regime $(\rho=0)$, the average per bond is $-J_{A F} S^{2}$. When $J_{A F}=0$, the spin background of the electron-poor regime can be ferromagnetic. The phase separation arises in a very small regime near the half-filling. For a finite $J_{A F}$, the spin background is antiferromagnetic. Hence the average energy per bond for the whole system in the phase separation is $\epsilon_{p s}=-\left(J_{A F} S^{2}\right.$ $\left.+2 v_{0} \rho\right)$, which is always lower than the energy in an antiferromagnetic state with a uniform density of electrons. This conclusion holds for any dimensional cases. ${ }^{20}$ Thus the phase separation occurs in the antiferromagnetic background. The phase diagram in Fig. 1 shows that the phase separation occurs in the case of either high or low density of electrons. Between the ferromagnetic phase and the phase separation, it is a paramagnetic or noncollinear magnetic regime. The phase diagram is in good agreement with those established by utilizing Monte-Carlo simulation by Yunoki et al. ${ }^{21}$ The phase separation always occurs near $\rho \rightarrow 1$ no matter how large $J_{A F}$ is and $v_{0}>0$ because the antiferromagnetic coupling is always predominant in the limit. This is quiet similar to those obtained in the $t$ - $J$ model in the large $S$ limit. ${ }^{22}$ Near $\rho \rightarrow 0, J_{A F}$ will determine whether the phase separation arises as the ferromagnetic coupling will dominate over the antiferromagnetic coupling if $J_{A F}=0$. When $J_{A F} \neq 0$, the phase separation can arise since the double exchange ferromagnetic coupling is approximately proportional to the density of electron near $\rho=0$, which is always less than a constant $J_{A F}$ at a sufficiently dilute doping. This is also consistent with the numerical results. ${ }^{21}$ For a fixed $v_{0}$, the phase separation can occur when $J_{A F}$ increases as shown in Fig. 2. If $v_{0}$ is very large, the phase separation can occur for any $J_{A F}$, and vice versa. If $v_{0}=0$, i.e., the strong Hund coupling, the phase separation does not arise. Thus the attraction plays a decisive role in the phase separation. It is worth mentioning that even in the paramagnetic phase the average value of attraction potential $v_{0}\left(\left\langle\cos \Theta_{i j}\right\rangle-1\right)=-v_{0}$, half of that in the antiferro- 
magnetic case. When the phase separation occurs, it will enhance the antiferromagnetic coupling especially in the electron-rich regime. This agrees with experimental observation that the charge stripe arises at higher temperature than the spin stripe by electron-diffraction and neutron-scattering measurements. 1

As a conclusion, we discuss possible relevances of the attraction, the phase separation, and the charge stripe phase. Although the attraction could attract the electrons together to form an electron-rich regime, other physical mechanism has to be taken into account in order to explain the stripe behaviors for charge and spin. Löw et al. proposed the ideas of frustrated phase separation by considering the nearestneighbor attraction and long-range Coulomb repulsion. ${ }^{6}$ Our work provides a direct evidence that an attraction between electrons indeed arises from the superexchange of electrons for the finite $J_{H}$ case. On the other hand, the role of hopping term is still unclear in forming the stripe phase. Recent numerical calculation by density-matrix renormalization group (DMRG) found some evidence that the stripe behaviors ap- pear in the two-dimensional $t-J$ model $^{23}$ for a specific dopant due to the electron hopping even without the Coulomb interaction. Our findings provide a direct mechanism for electrons to condensate along the charge stripe. For example, on a square lattice and at $\rho=2 / 3$, a static charge stripe as shown in the inset of Fig. 3 has a lower energy than the state with a uniform density of charge for a larger $v_{0}$. The larger $J_{A F} S^{2}$ is the lower the energy of the charge stripe is for a fixed $v_{0}$. However the stripe state is unstable against the phase separation. To stabilize the stripe state, one should consider other physical processes; for example, effect of long-range Coulomb interaction ${ }^{6}$ and noncollinear magnetism of localized spins. In short, since the spin background modulates the electronic behaviors, we believe that our model (5) or its quantum form (3) is a good starting point to investigate the phase separation, the charge ordering and spin ordering in doped transition-metal oxides.

This work was supported by a CRCG research grant at the University of Hong Kong.
${ }^{1}$ J. M. Tranquada et al., Nature (London) 375, 561 (1995); V. Sachan et al., Phys. Rev. B 54, 12318 (1996).

${ }^{2}$ S. M. Hayden et al., Phys. Rev. Lett. 68, 1061 (1992); C. H. Chen et al., ibid. 71, 2461 (1993).

${ }^{3}$ Z. Jirak et al., J. Magn. Magn. Mater. 53, 153 (1985); B. J. Sternlieb et al., Phys. Rev. Lett. 76, 2169 (1995); C. H. Chen and S.-W. Cheong, ibid. 76, 4042 (1996).

${ }^{4}$ G. Blumberg et al., Phys. Rev. Lett. 80, 564 (1998).

${ }^{5}$ Y. Tomioka et al., Phys. Rev. Lett. 74, 5108 (1995).

${ }^{6}$ U. Löw et al., Phys. Rev. Lett. 72, 1918 (1994).

${ }^{7}$ J. M. D. Coey et al., Phys. Rev. Lett. 75, 3910 (1995).

${ }^{8}$ P. Kuiper et al., Phys. Rev. Lett. 62, 221 (1989); H. Eisaki et al., Phys. Rev. B 45, 12513 (1992).

${ }^{9}$ C. Zener, Phys. Rev. 82, 403 (1951).

${ }^{10}$ P. W. Anderson and H. Hasegawa, Phys. Rev. 100, 675 (1955).

${ }^{11}$ P. G. de Gennes, Phys. Rev. 118, 141 (1960).

${ }^{12}$ J. R. Schrieffer and P. A. Wolff, Phys. Rev. 149, 491 (1966).

${ }^{13}$ K. Kubo and N. Ohata, J. Phys. Soc. Jpn. 33, 21 (1972).

${ }^{14}$ S. Q. Shen, Phys. Lett. A 235, 403 (1997).

${ }^{15}$ S. Q. Shen, Phys. Rev. B 53, 14252 (1996); 55, 14330 (1997).
${ }^{16}$ F. Dyson, E. H. Lieb, and B. Simon, J. Stat. Phys. 18, 335 (1978).

${ }^{17}$ V. J. Emery, S. A. Kivelson, and H. Q. Lin, Phys. Rev. Lett. 64, 475 (1990); for reviews, see S. A. Kivelson and V. J. Emery, in Strongly Correlated Electronic Materials: The Los Alamos Symposium, 1993, edited by K. S. Bedell et al. (Addison-Wesley, Reading, New York, 1994), p. 619 and E. Daggoto, Rev. Mod. Phys. 66, 763 (1994), and references therein.

${ }^{18}$ C. S. Hellberg and E. Manousakis, Phys. Rev. Lett. 78, 4609 (1997); C. T. Shih, Y. C. Chen, and T. K. Lee, Phys. Rev. B 57, 627 (1998).

${ }^{19}$ J. Inoue and S. Maekawa, Phys. Rev. Lett. 74, 3407 (1995).

${ }^{20}$ It should be pointed out that the ratio of the densities in the electron-poor and electron-rich regimes are not always 0 . We can say the phase separation occurs as far as the ratio deviates from 1.

${ }^{21}$ S. Yunoki et al., Phys. Rev. Lett. 80, 845 (1998); S. Yunoki and A. Moreo, Phys. Rev. B 58, 6403 (1998).

${ }^{22}$ A. Auerbach and B. E. Larson, Phys. Rev. B 43, 7800 (1991).

${ }^{23}$ S. R. White and D. J. Scalapino, Phys. Rev. Lett. 80, 1272 (1998). 